\title{
Determination of the biomechanical effect of an interspinous process device on implanted and adjacent lumbar spinal segments using a hybrid testing protocol: a finite-element study
}

\author{
Deniz U. Erbulut, PhD,, 1 Iman Zafarparandeh, MSc, ${ }^{1}$ Chaudhry R. Hassan, BSc, ${ }^{1}$ \\ Ismail Lazoglu, PhD, ${ }^{1}$ and Ali F. Ozer, MD² \\ Departments of ${ }^{1}$ Mechanical Engineering and ${ }^{2}$ Neurosurgery, Koc University, Istanbul, Turkey
}

OBJECT The authors evaluated the biomechanical effects of an interspinous process (ISP) device on kinematics and load sharing at the implanted and adjacent segments.

METHODS A 3D finite-element (FE) model of the lumbar spine (L1-5) was developed and validated through comparison with published in vitro study data. Specifically, validation was achieved by a flexible (load-control) approach in 3 main planes under a pure moment of $10 \mathrm{Nm}$ and a compressive follower load of $400 \mathrm{~N}$. The ISP device was inserted between the L-3 and L-4 processes. Intact and implanted cases were simulated using the hybrid protocol in all motion directions. The resultant motion, facet load, and intradiscal pressure after implantation were investigated at the index and adjacent levels. In addition, stress at the bone-implant interface was predicted.

RESULTS The hybrid approach, shown to be appropriate for adjacent-level investigations, predicted that the ISP device would decrease the range of motion, facet load, and intradiscal pressure at the index level relative to the corresponding values for the intact spine in extension. Specifically, the intradiscal pressure induced after implantation at adjacent segments increased by $39.7 \%$ and by $6.6 \%$ at $L 2-3$ and $L 4-5$, respectively. Similarly, facet loads at adjacent segments after implantation increased up to $60 \%$ relative to the loads in the intact case. Further, the stress at the bone-implant interface increased significantly. The influence of the ISP device on load sharing parameters in motion directions other than extension was negligible.

CONCLUSIONS Although ISP devices apply a distraction force on the processes and prevent further extension of the index segment, their implantation may cause changes in biomechanical parameters such as facet load, intradiscal pressure, and range of motion at adjacent levels in extension.

http://thejns.org/doi/abs/10.3171/2014.12.SPINE14419

KEY WORDS interspinous device; posterior stabilization; lumbar spine

$\mathrm{I}$ MPLANTATION of interspinous process (ISP) devices is known to be useful as a surgical treatment for different types of spinal pathologies, such as spinal stenosis or facet arthritis. ${ }^{1,4,26}$ The main design goal of such devices is to apply a distraction force to the processes and prevent further extension of the segment. As a result, ISP devices help to relieve the symptoms of neurogenic intermittent claudication associated with spinal stenosis. Further, these devices are designed to limit extension and expand the spinal column and foramen at the treated level. They are also designed to allow motion during flexion, lateral bending, and axial rotation at the treated segment because they reduce adjacent-level effects. ${ }^{4,6}$

Of all ISP devices, the X-Stop device (Saint Francis Medical Technologies Inc.) has been documented most extensively in the literature. ${ }^{20}$ It has 2 lateral wings to prevent unnecessary displacements. ${ }^{9}$ Its design is aimed mostly at preventing extension while allowing normal motion in other motion planes. In an in vitro study, Lindsey et al. ${ }^{12}$ investigated the effect of X-Stop implantation on the ki-

ABBREVIATIONS ALE = adjacent-level effect; FE = finite element; FN = flying node; IDP = intradiscal pressure; ISL = interspinous ligament; ISP = interspinous process; $\mathrm{ROM}=$ range of motion; $\mathrm{SSL}=$ supraspinous ligament.

ACCOMPANYING EDITORIAL See pp 197-199. DOI: 10.3171/2015.1.SPINE141277.

SUBMITTED August 11, 2014. ACCEPTED December 18, 2014.

INCLUDE WHEN CITING Published online May 1, 2015; DOI: 10.3171/2014.12.SPINE14419.

DISCLOSURE The authors report no conflict of interest concerning the materials or methods used in this study or the findings specified in this paper. 
nematics of implanted and adjacent segments and found that it significantly reduced the range of motion (ROM) in extension at the implanted level while retaining the $\mathrm{ROM}$ in flexion, axial rotation, and lateral bending. They also found that the device did not affect the ROM of the adjacent segments in any motion direction. In another in vivo study, Siddiqui et al. ${ }^{24}$ investigated the changes in disc height and segmental and total lumbar rotation before and after X-Stop implantation and concluded that the disc height and sagittal kinematics of the lumbar spine are not significantly affected by the device.

Wilke et al. ${ }^{26}$ compared the effects of 4 interspinous implants-Coflex (Paradigm Spine), Wallis (Abbot Spine), DIAM (Medtronic), and X-Stop-on ROM and intradiscal pressure (IDP) in an in vitro test in which pure moments were applied on a functional spinal unit in flexionextension, lateral bending, and axial rotation. Their results showed that all implants reduced ROM in extension by about $50 \%$ of that in the intact model. However, none of them affected ROM in other directions. Further, as was the case with ROM, all implants reduced IDP in extension but did not have any effect on it in other directions. In another in vitro study, Hartmann et al. ${ }^{6}$ studied the biomechanical effect of 4 interspinous implants-Aperius (Kyphon), In-Space (Synthes), X-Stop, and Coflex-on the ROM at implanted and adjacent levels using L1-5 cadaveric specimens. They found that all 4 devices caused a significant reduction in ROM in extension but did not affect it under pure moments in other directions. They also showed that when a follower load was applied with pure moments, the ROM in flexion decreased for all 4 implants. Wiseman et al. ${ }^{27}$ determined the effect of an implanted X-Stop device on the facet load in extension at the index and adjacent segments of L2 -5 cadaveric specimens and found a significant reduction in facet loads at the index level but no change in the loads at the adjacent level. Swanson et al. ${ }^{25}$ investigated the influence of X-Stop implantation on the IDP at the index (L3-4) and adjacent levels in vitro. They observed that the device did not significantly affect IDP at the adjacent level, although it was decreased considerably at the index level.

All the above-mentioned studies were in vitro; however, in vitro investigations have several limitations. For example, as reported by Wilke et al., ${ }^{26}$ the IDP in vitro may not be calculated correctly by using pressure transducers. However, finite-element (FE) studies, unlike in vitro studies, can help researchers to examine the inner workings of the lumbar spine and study the effects of an ISP device on load sharing, stresses, and strains in the spine under different loading conditions. To this end, Lafage et al. ${ }^{10}$ conducted a combined in vitro and FE study to investigate the effect of the Wallis implant on ROM and stress at the disc annulus and implant at the index and superior adjacent levels. They used the L3-5 segments for cadaver and FE studies and observed a reduction in motion and disc internal loads in the case of the implanted segment. However, their model was limited to studying the adjacent-segment effect since it had just 2 segments. Furthermore, they applied a flexible protocol (load control) instead of a hybrid protocol (displacement control). The latter is more anatomically relevant and can better represent the motion and loads experienced by the spine after surgical procedures and implantation. ${ }^{5,16}$ However, in the field of ISP devices, no study has utilized the FE approach for a detailed biomechanical investigation of the effect of these devices on adjacent segments by using a hybrid protocol.

Therefore, the aim of the present FE study was to evaluate the effect of an implanted ISP device on the biomechanical parameters of a lumbar spine by using a hybrid testing protocol. The biomechanical parameters considered were ROM, IDP, and facet load at both the index and the adjacent level. In addition, stress in the ISP device after implantation was evaluated.

\section{Methods \\ Intact Model}

We developed a 3D FE model of the L1-5 segments of the spine. The geometry of the vertebrae was obtained from CT scan data for a healthy 35-year-old man. The lordosis curvature was measured as $25^{\circ}$ using the Cobb method (Fig. 1 left). All cancellous, cortical, and posterior parts of the vertebrae were meshed using hexahedral elements (C3D8). The outer layer of the elements on each vertebra was considered as the cortical layer with a thickness of approximately $0.5 \mathrm{~mm} .{ }^{17}$ Further, the adjacent level of the disc was considered as the endplate with an average thickness of $0.6 \mathrm{~mm}$, and the cancellous part of the vertebrae composed the inner mesh. The detailed meshing procedure has been reported in our previous study. ${ }^{3}$ Threedimensional gap contact elements (GAPUNI) were used to simulate the facet joints between the vertebrae. These elements transfer force between nodes in a single direction as a function of the specified gap between them. The behavior of the cartilaginous layers of the facet surfaces was simulated with a parameter called "softened contact" in the ABAQUS software (Version 6.10-2, Abaqus Inc.).

The circular mesh pattern ${ }^{3}$ on the disc helped to model the concentric rings of the annulus ground substance. The rebar option of ABAQUS oriented $\pm 30^{\circ}$ to the horizontal plane was used to model the fibers in the annulus. The "no compression" option of the ABAQUS software was used to restrict the fibers only under tension loading. A hyperelas-

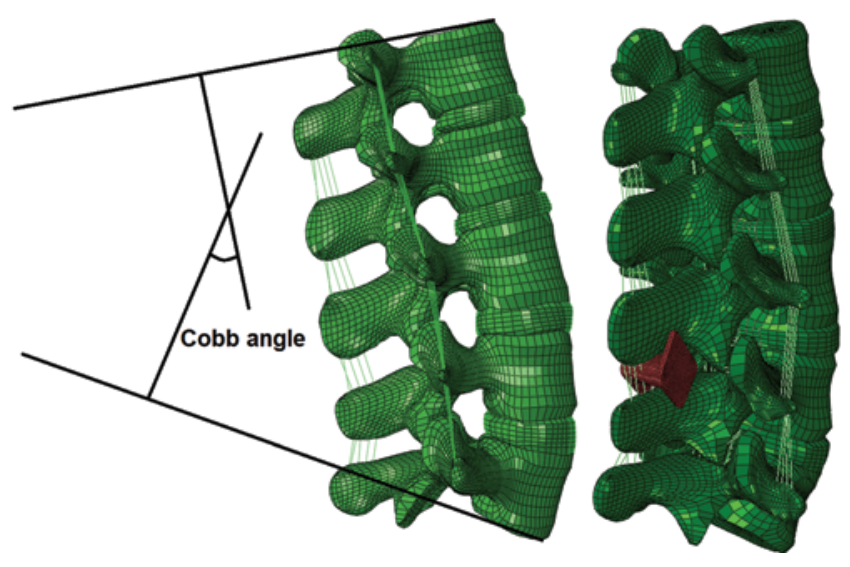

FIG. 1. Finite-element model of the lumbar spine $(L 1-5)$ implanted with an ISP device at the L3-4 segment. Figure is available in color online only. 
TABLE 1. Mechanical properties and element types of lumbar FE model components

\begin{tabular}{llcc}
\hline & \multicolumn{1}{c}{ Element Formulation } & Modulus in MPa (elongation) & Poisson's Ratio \\
\hline Component & & & 0.25 \\
\hline Vertebral cancellous bone & Isotropic, elastic hex elements & 450 & 0.3 \\
\hline Vertebral cortical bone & Isotropic, elastic hex elements & 12,000 & 0.25 \\
\hline Posterior bone & Isotropic, elastic hex elements & 3500 & 0.4999 \\
\hline Nucleus pulposus & Isotropic, elastic hex elements & 9 & 0.3 \\
\hline Annulus (ground) & Hyperelastic, neo-Hookean & $\mathrm{C} 10=0.3448, \mathrm{D} 1=0.3$ & 0.3 \\
\hline Annulus (fiber) & Rebar & $357-550$ & 0.3 \\
\hline Ligament & & & 0.3 \\
\hline Anterior longitudinal & Truss elements & $7.8(<12 \%), 20.0(>12 \%)$ & 0.3 \\
\hline Posterior longitudinal & Truss elements & $10.0(<11 \%), 20.0(>11 \%)$ & 0.3 \\
\hline Ligamentum flavum & Truss elements & $10.0(<18 \%), 58.7(>18 \%)$ & 0.3 \\
\hline Intertransverse & Truss elements & $10.0(<14 \%), 11.6(>14 \%)$ & \\
\hline Interspinous & Truss elements & $8.0(<20 \%), 15.0(>20 \%)$ & \\
\hline Supraspinous & Truss elements & $7.5(<25 \%), 32.9(25 \%)$ & \\
\hline Capsular & Truss elements & & \\
\hline Apophyseal joints & GAPUNI & & \\
\hline
\end{tabular}

C10, D1 = components of a special tensor; GAPUNI = gap contact elements; hex = hexahedral.

tic material model was used to simulate behavior of the annulus. Further, fluid behavior of the nucleus was simulated using a hexahedral element assigned very low stiffness (1 $\mathrm{MPa}$ ) and near incompressibility (Poisson's ratio 0.4999).

The ligaments were simulated using 3D truss elements, which were constrained to act nonlinearly only in tension. All 7 major ligaments - that is, the anterior longitudinal ligament, posterior longitudinal ligament, ligamentum flavum, intertransverse ligament, interspinous ligament (ISL), supraspinous ligament (SSL), and capsular ligament-were represented. The ligaments' behavior, namely, the change in their stiffness with strain, was simulated using a hypoelastic material model.

The complete model consisted of 72,193 nodes and 55,650 elements that represented the entire structure of the lumbar spine (Fig. 1 right). The material properties of various components of the lumbar model (Table 1) were obtained from the literature.

\section{Boundary and Loading Conditions}

In all directions, the nodes lying on the upper endplate of L-1 were coupled to a flying node (FN) higher than the surface of the L-1 endplate; then a pure moment was applied to the FN. The follower load was applied on each side of all segments such that the unwanted segmental rotation was less than $0.2^{\circ} .{ }^{16}$ The follower load was simulated using the connector elements between each set of adjacent vertebrae. The nodes lying at the bottom endplate of L-5 were constrained in all directions.

The intact L1-5 lumbar model was validated in 3 motion planes against published in vitro study data. The amount of rotation in each segment was compared with that reported in published in vitro studies. A pure moment of $10 \mathrm{Nm}$ combined with an applied follower load of 400 $\mathrm{N}$ was used to simulate the model in flexion-extension and axial rotation, and the motion response was compared with those reported in the in vitro studies by Yamamoto et al. ${ }^{28}$ Schmoelz et al., ${ }^{22}$ Niosi et al., ${ }^{14}$ and Schilling et al. ${ }^{21}$

\section{Implanted Model}

An ISP device was inserted between the ISPs of the L-3 and L-4 segments; the design of this device, which was the static kind, was similar to X-Stop in that the cross-section of the device was oval (Fig. 1 right). The device had a core that could be accommodated between the processes without any injury to the ISL and SSL. Two wings were designed to keep the device in place. The upper elements of the device were coupled to adjacent elements on the processes of the corresponding vertebra. Titanium alloy was selected as the material for the device. The isotropic elastic formulation was used to simulate the material properties of titanium (Young's modulus $115 \mathrm{GPa}$, Poisson's ratio 0.3 ).

The implanted model was loaded in all 3 main directions: flexion-extension, lateral bending, and axial rotation. A hybrid protocol, which was used in all simulations, was implemented with the aim of examining adjacent-segment biomechanics by varying the moment until the overall deflection of the implanted L1-5 models equaled the predicted deflection for the intact model..$^{15,16}$ The hybrid moment for the implanted model was $14.5 \mathrm{Nm}$ in extension. A bending moment of $10 \mathrm{Nm}$ was used in all other directions because similar total ROMs were obtained in other motion directions.

\section{Results \\ Model Validation}

The FE model of the intact L1-5 segments was validated against reported in vitro studies ${ }^{14,21,22,28}$ in flexionextension, axial rotation, and lateral bending. A precompression load of $400 \mathrm{~N}$ and pure moment of $10 \mathrm{Nm}$ were 
TABLE 2. Ranges of motion predicted by the intact FE model compared with reported ROMs from in vitro studies*

\begin{tabular}{|c|c|c|c|c|c|}
\hline Authors \& Year & Pure Moment (Nm) & Flexion & Extension & Lateral Bending & Axial Rotation \\
\hline \multicolumn{6}{|l|}{ L1-2 } \\
\hline Yamamoto et al., 1989 & 10 & $5.8 \pm 0.6$ & $4.3 \pm 0.5$ & $\begin{array}{l}4.7 \pm 0.4(\mathrm{lt}) \\
5.2 \pm 0.4(\mathrm{rt})\end{array}$ & $\begin{array}{l}2.6 \pm 0.5(\mathrm{lt}) \\
2.0 \pm 0.6(\mathrm{rt})\end{array}$ \\
\hline Present study & 10 & 3.4 & 4.1 & $\begin{array}{l}7.0(\mathrm{lt}) \\
7.1(\mathrm{rt})\end{array}$ & $\begin{array}{l}3.9(\mathrm{lt}) \\
3.5(\mathrm{rt})\end{array}$ \\
\hline \multicolumn{6}{|l|}{ L2-3 } \\
\hline Schmoelz et al., 2003 & 10 & $4.3 \pm 1.0$ & $4.6 \pm 2.2$ & $5.4 \pm 2.2$ & $1.0 \pm 1.0$ \\
\hline Yamamoto et al., 1989 & 10 & $6.5 \pm 0.3$ & $4.3 \pm 0.3$ & $\begin{array}{l}7.0 \pm 0.6(\mathrm{lt}) \\
7.0 \pm 0.6(\mathrm{rt})\end{array}$ & $\begin{array}{l}2.2 \pm 0.4(\mathrm{lt}) \\
3.0 \pm 0.4(\mathrm{rt})\end{array}$ \\
\hline Present study & 10 & 4.1 & 2.9 & $\begin{array}{l}6.0(\mathrm{lt}) \\
6.7(\mathrm{rt})\end{array}$ & $\begin{array}{l}3.2(\mathrm{lt}) \\
3.4(\mathrm{rt})\end{array}$ \\
\hline \multicolumn{6}{|l|}{ L3-4 } \\
\hline Niosi et al., 2006 & 7.5 & $4.4 \pm 2.0$ & $2.4 \pm 0.9$ & $2.4 \pm 1.2$ & $1.2 \pm 0.5$ \\
\hline Schilling et al., 2011 & 7.5 & $4.67 \pm 1.79$ & $2.18 \pm 0.54$ & $7.66 \pm 2.91$ & $4.67 \pm 2.52$ \\
\hline Schmoelz et al., 2003 & 10 & $5.0 \pm 1.0$ & $4.0 \pm 1.3$ & $4.7 \pm 2.0$ & $1.0 \pm 0.6$ \\
\hline Yamamoto et al., 1989 & 10 & $7.5 \pm 0.8$ & $3.7 \pm 0.3$ & $\begin{array}{l}5.7 \pm 0.3(\mathrm{lt}) \\
5.8 \pm 0.5(\mathrm{rt})\end{array}$ & $\begin{array}{l}2.7 \pm 0.4(\mathrm{lt}) \\
2.5 \pm 0.4(\mathrm{rt})\end{array}$ \\
\hline Present study & 10 & 3.7 & 3.7 & $\begin{array}{l}6.0(\mathrm{lt}) \\
7.5(\mathrm{rt})\end{array}$ & $\begin{array}{l}3.7(\mathrm{lt}) \\
3.8(\mathrm{rt})\end{array}$ \\
\hline \multicolumn{6}{|l|}{ L4-5 } \\
\hline Schilling et al., 2011 & 7.5 & $5.62 \pm 2.17$ & $3.32 \pm 1.12$ & $7.76 \pm 1.85$ & $5.16 \pm 1.30$ \\
\hline Yamamoto et al., 1989 & 10 & $8.9 \pm 0.7$ & $5.8 \pm 0.4$ & $\begin{array}{l}5.5 \pm 0.5(\mathrm{lt}) \\
5.9 \pm 0.5(\mathrm{rt})\end{array}$ & $\begin{array}{l}1.7 \pm 0.3(\mathrm{lt}) \\
2.7 \pm 0.5(\mathrm{rt})\end{array}$ \\
\hline Present study & 10 & 5.5 & 3.5 & $\begin{array}{l}6.7(\mathrm{lt}) \\
7.3(\mathrm{rt})\end{array}$ & $\begin{array}{l}4.1(\mathrm{It}) \\
4.0(\mathrm{rt})\end{array}$ \\
\hline
\end{tabular}

* Values expressed in degrees, unless indicated otherwise.

applied to the FE model to predict the ROM values. Table 2 lists results of a comparison between the motion values predicted by the FE model and those reported in published in vitro studies. The kinematic data predicted by the FE model were within the standard deviation or close to the average of the cadaveric data obtained from the literature. The small variations in the ROMs between the studies were observed only at the L1-2 segment in flexion and axial rotation; these variations were attributed to differences in the methods and loading conditions used in the cadaveric studies. In the present study, we used this validated FE model for evaluating the effect of ISP devices under hybrid loading in extension. A comparison of the ROM values at the L1-2 segment predicted by the FE model with those obtained in the in vitro study by Yamamoto et al. ${ }^{28}$ showed that the FE model-predicted values in extension and axial rotation were in the range of those found in the in vitro study. In lateral bending and flexion, however, the ROM values were $25 \%$ higher and $41 \%$ lower, respectively, than the values in the in vitro study. ${ }^{28}$ In other words, the FE model-predicted motion values were lower in flexion but higher in lateral bending and axial rotation as compared with the corresponding in vitro values.

\section{Range of Motion}

Figure 2 shows the ROM at each lumbar level for both the intact and implanted models in flexion, extension, lat- eral bending, and axial rotation. In the extension direction, ROM at the L3-4 segment decreased by $80.6 \%$ after implantation relative to the ROM at this segment in the intact lumbar spine. Further, the ROM values in adjacent segments-that is, L2-3 and L4-5-increased by $29.7 \%$ and $21 \%$, respectively, relative to those in the corresponding segments in the intact spine.

At the index level, ROM changed by up to $8 \%$ and $3 \%$ in lateral bending and axial rotation, respectively, but it did not change in flexion. At the adjacent level, the change in the ROM was the same-by up to $2 \%$-in flexion, lateral bending, and axial rotation.

\section{Facet Loads}

Table 3 lists the left and right facet loads at the index and adjacent levels. In extension, a comparison of facet loads at the index and adjacent levels in both models reveals that at the index level in the implanted model, the facet loads decreased significantly - up to $99.9 \%$-relative to the intact model. However, at the superior (L2-3) and inferior (L4-5) adjacent levels, the facet loads increased up to $51.9 \%$ and up to $60.3 \%$, respectively.

At the index level, the facet load changed by up to $22 \%$ and $15 \%$ in lateral bending and axial rotation, respectively, but it did not change in flexion. At the adjacent level, the change in the facet load was the same-by up to $7 \%$-in flexion, lateral bending, and axial rotation. 

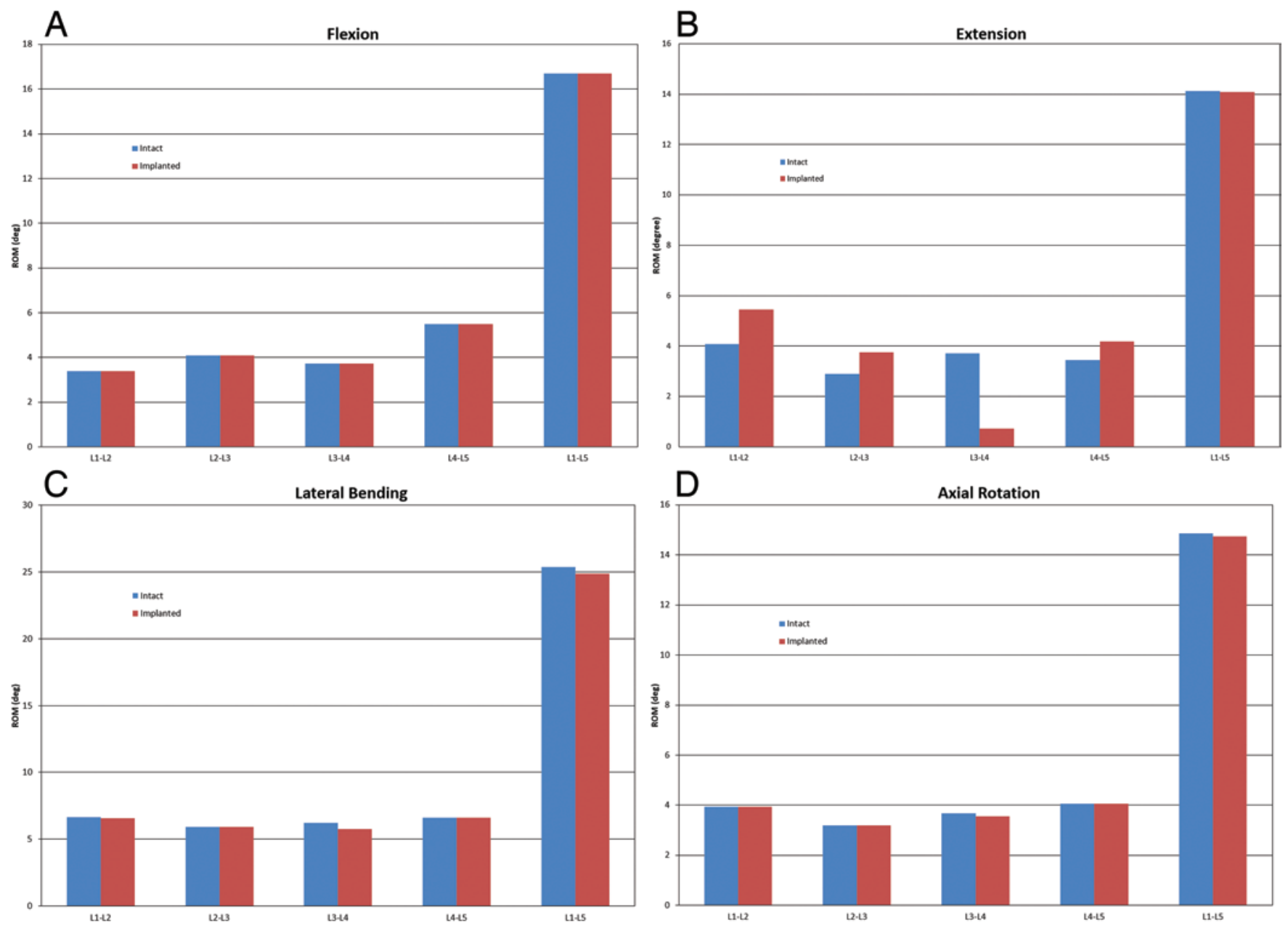

FIG. 2. Range of motion for the intact and implanted lumbar models. Pure bending moment of $10 \mathrm{Nm}$ and follower load of $400 \mathrm{~N}$ were applied to the intact model in 3 main planes. Hybrid moment of $14.5 \mathrm{Nm}$ and follower load of $400 \mathrm{~N}$ applied to the implanted model in extension and bending moment of $10 \mathrm{Nm}$ and follower load of $400 \mathrm{~N}$ applied to the implanted model in flexion, lateral bending, and axial rotation. Figure is available in color online only.

\section{Intradiscal Pressure}

Table 4 presents the maximum IDP at the index and adjacent levels for both the intact and implanted models. The predicted IDP at the index level in the intact model was $0.98 \mathrm{MPa}$ in extension but it decreased by $52.6 \%$ after implantation. In contrast, the predicted IDP at the superior adjacent level in the intact model was $0.87 \mathrm{MPa}$ but it increased significantly by $40 \%$ after implantation. Further, the predicted IDP at the inferior adjacent level in the intact model was $0.90 \mathrm{MPa}$ but it increased moderately by $6.6 \%$ after implantation.

At the index level, the IDP changed by $5 \%$ in lateral bending, but it did not change in flexion and axial rotation. At the adjacent level, the IDP was the same as that in the intact model in flexion, lateral bending, and axial rotation.

\section{Stress in Spinous Processes}

Figure 3 shows the predicted von Mises stress distribution in spinous processes before and after implantation, in extension. The maximum von Mises stress in the L-3 spinous process increased significantly (to $53 \mathrm{MPa}$ ) after implantation. Similarly, the maximum predicted von Mises stress in the L-4 spinous process was $48.2 \mathrm{MPa}$ after implantation.

\section{Discussion}

Interspinous process devices are useful in the treatment of various lumbar spine pathologies. Several studies have investigated the effects of such devices on the biomechanical behavior of the lumbar spine at both the index and adjacent segments. However, none of these studies applied the hybrid protocol to investigate adjacent-segment effects. As suggested by Panjabi et al., ${ }^{15,16}$ a hybrid approach gives results that represent the actual scenario in clinical cases following surgical procedures and implantation. In particular, a hybrid testing protocol is appropriate for the biomechanical evaluation of adjacent spinal levels. ${ }^{16}$ Studies have shown that the hybrid protocol is more anatomically relevant and can better represent the motion and loads experienced by the spine after surgical procedures and implantation..$^{5,16}$ Therefore, we attempted to evaluate 
TABLE 3. Comparison of predicted facet load between the intact and instrumented FE models for the index and adjacent segments*

\begin{tabular}{|c|c|c|c|c|c|c|c|c|c|c|c|c|}
\hline \multirow[b]{2}{*}{ Condition } & \multicolumn{3}{|c|}{ Flexion } & \multicolumn{3}{|c|}{ Extension } & \multicolumn{3}{|c|}{ Lateral Bending } & \multicolumn{3}{|c|}{ Axial Rotation } \\
\hline & L2-3 & L3-4 & L4-5 & L2-3 & L3-4 & L4-5 & L2-3 & L3-4 & L4-5 & L2-3 & L3-4 & L4-5 \\
\hline \multicolumn{13}{|l|}{ Intact } \\
\hline $\mathrm{Rt}$ & 45.63 & 76.68 & 25.89 & 127.13 & 147.36 & 120.37 & 29.40 & 54.67 & 76.17 & 0 & 0 & 0 \\
\hline Lt & 54.40 & 77.44 & 19.46 & 151.72 & 156.99 & 194.33 & 37.51 & 59.35 & 21.93 & 172.11 & 175.72 & 214.00 \\
\hline \multicolumn{13}{|l|}{ Implanted } \\
\hline Rt & 45.66 & 76.68 & 25.89 & 184.02 & 0.06 & 192.95 & 29.84 & 66.32 & 73.95 & 0 & 0 & 0 \\
\hline $\mathrm{Lt}$ & 54.40 & 77.44 & 19.46 & 230.38 & 11.06 & 299.92 & 37.53 & 46.12 & 23.55 & 172.16 & 150.12 & 215.17 \\
\hline
\end{tabular}

* Values expressed in $\mathrm{N}$.

the biomechanical effect of ISP devices on adjacent and index segments by using the hybrid protocol, which has rarely been attempted in this field.

The FE model (L1-5) used in this study was successfully validated in all 3 main planes via a comparison of its results with those from various published in vitro studies. Specifically, the model was validated given the fact that the kinematic data predicted by the FE model in all segments were within the standard deviation or close to the average of the results from in vitro studies.

According to the literature, ${ }^{1,18,26}$ all commercially available ISP devices affect the biomechanics of the lumbar spine only in extension but not in flexion, lateral bending, and axial rotation. Therefore, we modeled an elliptical cylinder-shaped device with lateral wings that mimicked the X-Stop device and placed it between the L-3 and L-4 spinous processes of the FE model (L1-5).

We simulated the implanted model in all motion directions. In general, changes in the ROM, IDP, and facet loads were negligible in directions other than extension, as reported in the literature. ${ }^{6,26}$ As expected, the IDP, facet load, and ROM decreased after ISP implantation in extension at the index level.

Our kinematic results at the index level in extension were in agreement with corresponding results in the literature. ${ }^{6,10,12,26}$ Specifically, our FE study predicted that the ROM reduced to $0.72^{\circ}$ after implantation at the index level in extension, which is similar to Lindsey et al.'s ${ }^{12}$ observed reduction of $0.5^{\circ} \pm 0.3^{\circ}$ after ISP implantation at the index level in extension. Another study ${ }^{6}$ reported similar results, that is, a reduction in extension, after ISP implantation in flexion-extension. Although we applied the hybrid protocol, which leads to higher loading $(14.5 \mathrm{Nm})$, the restriction of motion in extension at the implanted level was almost the same as that in the aforementioned studies, owing to the design of ISP devices that makes them restrict motion in extension.

The question then is whether ISP surgery affects the kinematics of adjacent segments. Lindsey et al., ${ }^{12}$ who had determined ROM at adjacent levels (L2-3 and L4-5) after implantation of the X-Stop ISP device into a cadaveric lumbar spine (L2-5), reported that the ROM at adjacent segments was not significantly affected in flexion-extension. Similarly, Hartmann et al. ${ }^{6}$ reported that there was no significant change in the ROM for all segments (L2-5) in flexion-extension under a pure moment of $7.5 \mathrm{Nm}$ and follower load of $400 \mathrm{~N}$. However, they reported a significant increase in the ROM for an entire specimen (L2-5) during lateral bending and rotation after implantation of 4 different ISP devices, and they suggested further investigation of ISP devices for determining adjacent-level effects (ALEs).

In contrast to these findings, our FE model predicted increased motion in the superior and inferior adjacent segments after ISP implantation in extension under hybrid loading (pure moment of $14.5 \mathrm{Nm}$ ) and a follower load of $400 \mathrm{~N}$. Although an increase in motion may not lead to adjacent-segment hypermobility due to changes in extension only and perhaps is not clinically significant, it may be useful to investigate the effect of motion changes on other components at adjacent levels, such as facet joints and intervertebral discs.

Further, in this study the facet loads predicted by the FE model for the intact model at the index segment were consistent with those reported in the literature. ${ }^{7}$ After implantation of the ISP device, the facet load decreased at the index level in extension. Wiseman et al. ${ }^{27}$ reported similar findings: they reported ALEs after implantation and found an increase in the facet load (by 10\%) at the superior adjacent level and a decrease in the facet load (by 17\%) at the inferior adjacent level. However, they also reported

TABLE 4. Comparison of predicted IDP between the intact and instrumented FE models for the index and adjacent segments*

\begin{tabular}{|c|c|c|c|c|c|c|c|c|c|c|c|c|}
\hline \multirow[b]{2}{*}{ Condition } & \multicolumn{3}{|c|}{ Flexion } & \multicolumn{3}{|c|}{ Extension } & \multicolumn{3}{|c|}{ Lateral Bending } & \multicolumn{3}{|c|}{ Axial Rotation } \\
\hline & L2-3 & L3-4 & L4-5 & L2-3 & L3-4 & L4-5 & L2-3 & L3-4 & L4-5 & L2-3 & L3-4 & L4-5 \\
\hline Intact & 1.44 & 1.34 & 1.46 & 0.87 & 0.98 & 0.90 & 2.04 & 1.80 & 2.11 & 1.34 & 1.33 & 1.22 \\
\hline Implanted & 1.44 & 1.34 & 1.46 & 1.21 & 0.47 & 0.96 & 2.04 & 1.7 & 2.11 & 1.34 & 1.33 & 1.22 \\
\hline
\end{tabular}

* Values expressed in MPa. 


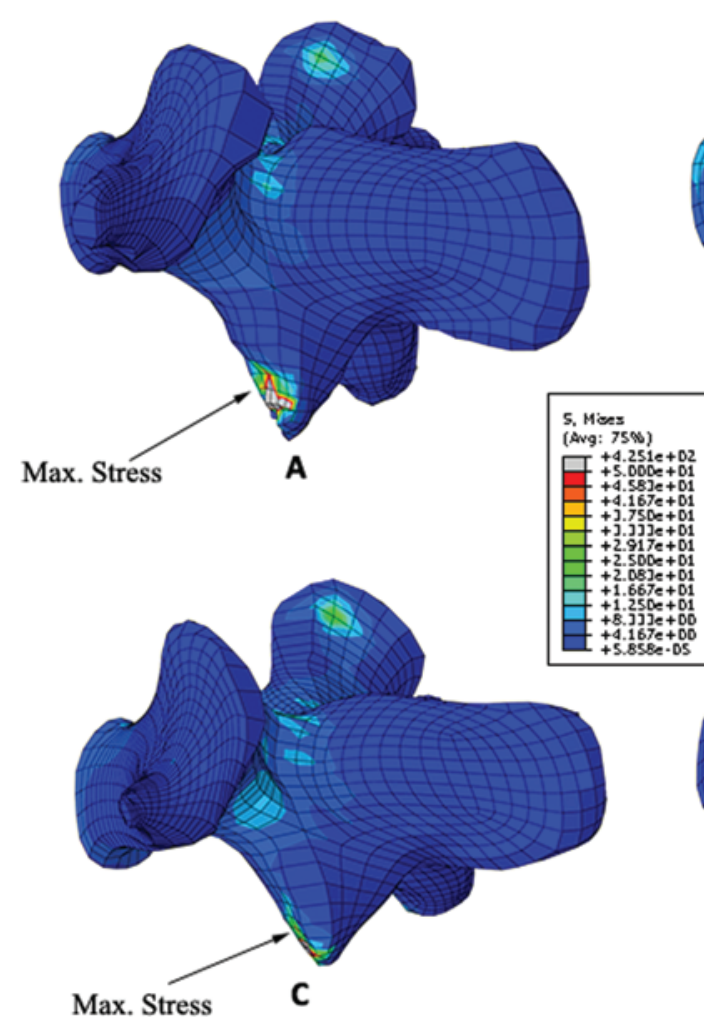

Intact
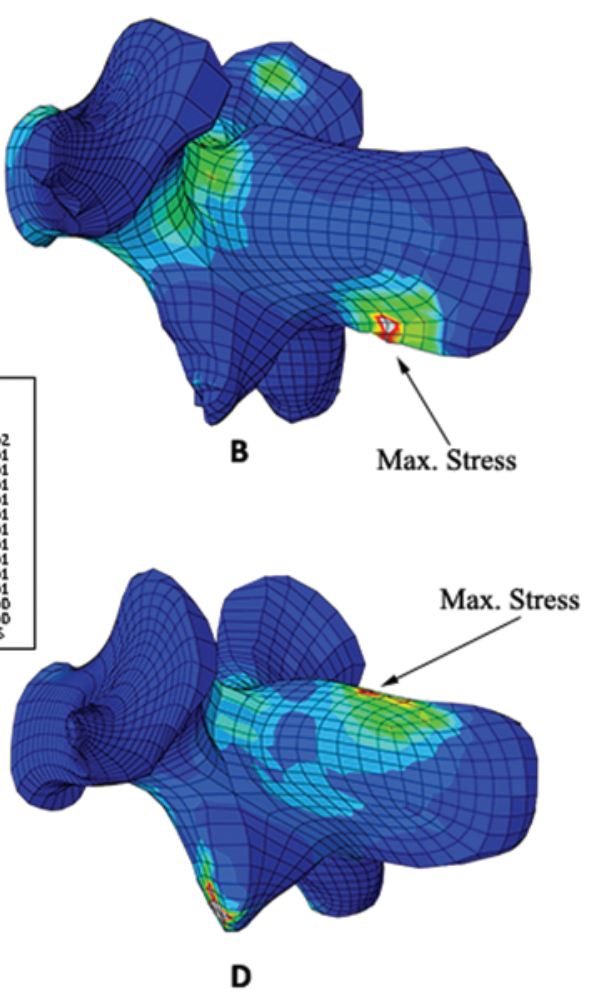

Instrumented

FIG. 3. Von Mises stress distribution at the posterior lumbar spine. A: Intact L-3 posterior. B: Implanted L-3 posterior. C: Intact L-4 posterior. D: Implanted L-4 posterior. Figure is available in color online only.

that the changes were not significantly different between the intact and implanted specimens. In contrast to their results, our FE model predicted that the facet load would increase by up to $60 \%$ in extension at both the superior and inferior adjacent levels. This contrast in results can be explained as follows. Wiseman et al. ${ }^{27}$ placed a pressuresensitive film in the facet joint to investigate the load values and compared them between the intact and implanted specimens at the adjacent and index segments by using a load control protocol. However, this approach has some drawbacks: $a \pm 15 \%$ error, which was associated with the pressure measurement; variation in the contact area of the pressure sensor; and unsuitability of the load control protocol for adjacent-level investigations. In comparison, the hybrid protocol is more appropriate for evaluating ALEs after implantation of a healthy lumbar FE model. Therefore, our results suggest that after ISP implantation, the adjacent-level facet joint will be affected over the long term and should be subjected to further investigation. At the index level, the facet loads changed by up to $22 \%$ and $15 \%$ in lateral bending and axial rotation, respectively. These changes were attributed to the wings of the ISP device, which limit motion in these directions. However, the total facet load in the index segment remained similar to that in the intact model, and only the load sharing between the right and left facets changed. In contrast, in extension, the total facet load changed-that is, it decreased.

Interspinous process devices prevent motion in extension and unload the intervertebral disc, resulting in an en- largement of the central canal for the treatment of neurogenic intermittent claudication. As expected, the FE model predicted that the ISP device would release IDP at the index level in extension. This result was in agreement with those in cadaveric studies in the literature. ${ }^{25,26}$ However, the FE model predicted that the IDP at the superior adjacent segment (L2-3) would increase by almost $40 \%$ after ISP implantation and that this IDP was very close to that in the intact case at the inferior adjacent segment (L4-5) in extension. Unfortunately, these results disagree with those in a cadaveric study by Swanson et al. ${ }^{25}$ One of the reasons for this disagreement is that Swanson and colleagues performed load control (that is, a flexibility test) instead of displacement control (that is, a hybrid test) for adjacentlevel investigations. Another reason, as mentioned in the previous paragraph when discussing the results for facet loads, is that Swanson et al. used a pressure transducer, which may not measure IDP correctly, as suggested by Wilke et al. ${ }^{26}$ Therefore, the efficiency of the intended use of ISP devices may not be correctly interpreted by flexible testing.

Although successful outcomes pertaining to the use of ISP devices in the treatment of lumbar spine diseases have been reported, ${ }^{11,29}$ a few cases of ISP failure after surgery have been described as well. For example, Miller et al. ${ }^{13}$ reported 2 cases of ISP failure in which gradual erosion of the spinous processes occurred because of consistent motion at the spacer-bone interface. Furthermore, Bowers et al. ${ }^{2}$ published medical records of complications associated 
with implantation of the X-Stop device. They reported the fracture of spinous processes in 3 of 13 patients and suggested possible causes of fracture, including the degree of osteoporosis and over-distraction of the interspinous space with an oversized implant. Kim et al. ${ }^{8}$ reported a high rate $(52 \%)$ of spinous process fracture associated with ISP surgery performed in 39 patients at their institution. One of the main reasons for spinous process fracture would be the stress concentration at the bone after ISP implantation. Our FE study drew a clear conclusion in this regard and showed that the stress distribution in spinous processes at the index level changed significantly after implantation. In the intact model, most of the stresses were concentrated at the facet joints. After implantation, however, a greater part of the stress was transferred to the implanted region (Fig. 3). Similarly, other studies ${ }^{10}$ reported an increase in the load transmitted through spinous processes. Although spinous processes may be strong enough to withstand the stresses after implantation, ${ }^{23}$ ISP devices may cause gradual erosion at the bone-implant interface under constant stress and motion, thereby increasing the recurrence probability of preoperative symptoms. In addition, the success rate of the outcome of lumbar treatment with an ISP device also depends largely on the bone density ${ }^{8}$ and overall lumbar scoliosis ${ }^{19}$ of patients.

Like any other numerical study, this study has certain limitations. Unlike cadaver studies, FE models do not account for the variation in material properties or geometry. Furthermore, some assumptions and simplifications in the model may not represent real values for the human spine. Nevertheless, the predicted data for the intact case are in agreement with the results of cadaver studies.

\section{Conclusions}

In this FE study, we analyzed the effect of an ISP device on biomechanical parameters of the lumbar spine using a hybrid protocol. The FE model predicted that the IDP, facet load, and ROM would decrease after ISP implantation at the index segment in extension. However, the FE model also predicted that the facet loads would increase in extension at both the superior and inferior adjacent levels. In addition, IDP increased at the superior adjacent segment. The FE model also showed that the stress distribution in spinous processes at the index level would increase significantly after implantation, which might cause gradual erosion at the bone-implant interface under constant stress and motion. Overall, our data suggested that ISP devices should be subjected to further investigation to understand their long-term effects on lumbar spine biomechanics.

\section{References}

1. Bono CM, Vaccaro AR: Interspinous process devices in the lumbar spine. J Spinal Disord Tech 20:255-261, 2007

2. Bowers C, Amini A, Dailey AT, Schmidt MH: Dynamic interspinous process stabilization: review of complications associated with the X-Stop device. Neurosurg Focus 28(6):E8, 2010

3. Erbulut DU, Zafarparandeh I, Lazoglu I, Ozer AF: Application of an asymmetric finite element model of the C2-T1 cervical spine for evaluating the role of soft tissues in stability. Med Eng Phys 36:915-921, 2014
4. Erbulut DU, Zafarparandeh I, Ozer AF, Goel VK: Biomechanics of posterior dynamic stabilization systems. Adv Orthop 2013:451956, 2013

5. Goel VK, Grauer JN, Patel TC, Biyani A, Sairyo K, Vishnubhotla S, et al: Effects of charité artificial disc on the implanted and adjacent spinal segments mechanics using a hybrid testing protocol. Spine (Phila Pa 1976) 30:2755-2764, 2005

6. Hartmann F, Dietz SO, Hely H, Rommens PM, Gercek E: Biomechanical effect of different interspinous devices on lumbar spinal range of motion under preload conditions. Arch Orthop Trauma Surg 131:917-926, 2011

7. Kiapour A, Ambati D, Hoy RW, Goel VK: Effect of graded facetectomy on biomechanics of Dynesys dynamic stabilization system. Spine (Phila Pa 1976) 37:E581-E589, 2012

8. Kim DH, Shanti N, Tantorski ME, Shaw JD, Li L, Martha JF, et al: Association between degenerative spondylolisthesis and spinous process fracture after interspinous process spacer surgery. Spine J 12:466-472, 2012

9. Kondrashov DG, Hannibal M, Hsu KY, Zucherman JF: Interspinous process decompression with the X-STOP device for lumbar spinal stenosis: a 4-year follow-up study. J Spinal Disord Tech 19:323-327, 2006

10. Lafage V, Gangnet N, Sénégas J, Lavaste F, Skalli W: New interspinous implant evaluation using an in vitro biomechanical study combined with a finite-element analysis. Spine (Phila Pa 1976) 32:1706-1713, 2007

11. Lee J, Hida K, Seki T, Iwasaki Y, Minoru A: An interspinous process distractor (X STOP) for lumbar spinal stenosis in elderly patients: preliminary experiences in 10 consecutive cases. J Spinal Disord Tech 17:72-78, 2004

12. Lindsey DP, Swanson KE, Fuchs P, Hsu KY, Zucherman JF, Yerby SA: The effects of an interspinous implant on the kinematics of the instrumented and adjacent levels in the lumbar spine. Spine (Phila Pa 1976) 28:2192-2197, 2003

13. Miller JD, Miller MC, Lucas MG: Erosion of the spinous process: a potential cause of interspinous process spacer failure. J Neurosurg Spine 12:210-213, 2010

14. Niosi CA, Zhu QA, Wilson DC, Keynan O, Wilson DR, Oxland TR: Biomechanical characterization of the three-dimensional kinematic behaviour of the Dynesys dynamic stabilization system: an in vitro study. Eur Spine J 15:913-922, 2006

15. Panjabi M, Malcolmson G, Teng E, Tominaga Y, Henderson G, Serhan H: Hybrid testing of lumbar CHARITE discs versus fusions. Spine (Phila Pa 1976) 32:959-967, 2007

16. Panjabi MM: Hybrid multidirectional test method to evaluate spinal adjacent-level effects. Clin Biomech (Bristol, Avon) 22:257-265, 2007

17. Panjabi MM, Chen NC, Shin EK, Wang JL: The cortical shell architecture of human cervical vertebral bodies. Spine (Phila Pa 1976) 26:2478-2484, 2001

18. Richards JC, Majumdar S, Lindsey DP, Beaupré GS, Yerby SA: The treatment mechanism of an interspinous process implant for lumbar neurogenic intermittent claudication. Spine (Phila Pa 1976) 30:744-749, 2005

19. Rolfe KW, Zucherman JF, Kondrashov DG, Hsu KY, Nosova E: Scoliosis and interspinous decompression with the XSTOP: prospective minimum 1-year outcomes in lumbar spinal stenosis. Spine J 10:972-978, 2010

20. Sangiorgio SN, Sheikh H, Borkowski SL, Khoo L, Warren $\mathrm{CR}$, Ebramzadeh E: Comparison of three posterior dynamic stabilization devices. Spine (Phila Pa 1976) 36:E1251E1258, 2011

21. Schilling C, Krüger S, Grupp TM, Duda GN, Blömer W, Rohlmann A: The effect of design parameters of dynamic pedicle screw systems on kinematics and load bearing: an in vitro study. Eur Spine J 20:297-307, 2011

22. Schmoelz W, Huber JF, Nydegger T, Dipl-Ing, Claes L, Wilke HJ: Dynamic stabilization of the lumbar spine and its effects 
on adjacent segments: an in vitro experiment. J Spinal Disord Tech 16:418-423, 2003

23. Shepherd DE, Leahy JC, Mathias KJ, Wilkinson SJ, Hukins DW: Spinous process strength. Spine (Phila Pa 1976) 25:319-323, 2000

24. Siddiqui M, Karadimas E, Nicol M, Smith FW, Wardlaw D: Effects of X-STOP device on sagittal lumbar spine kinematics in spinal stenosis. J Spinal Disord Tech 19:328-333, 2006

25. Swanson KE, Lindsey DP, Hsu KY, Zucherman JF, Yerby SA: The effects of an interspinous implant on intervertebral disc pressures. Spine (Phila Pa 1976) 28:26-32, 2003

26. Wilke HJ, Drumm J, Häussler K, Mack C, Steudel WI, Kettler A: Biomechanical effect of different lumbar interspinous implants on flexibility and intradiscal pressure. Eur Spine J 17:1049-1056, 2008

27. Wiseman CM, Lindsey DP, Fredrick AD, Yerby SA: The effect of an interspinous process implant on facet loading during extension. Spine (Phila Pa 1976) 30:903-907, 2005

28. Yamamoto I, Panjabi MM, Crisco T, Oxland T: Three-dimensional movements of the whole lumbar spine and lumbosacral joint. Spine (Phila Pa 1976) 14:1256-1260, 1989
29. Zucherman JF, Hsu KY, Hartjen CA, Mehalic TF, Implicito DA, Martin MJ, et al: A multicenter, prospective, randomized trial evaluating the X STOP interspinous process decompression system for the treatment of neurogenic intermittent claudication: two-year follow-up results. Spine (Phila Pa 1976) 30:1351-1358, 2005

\section{Author Contributions}

Conception and design: Erbulut, Ozer. Acquisition of data: Erbulut, Zafarparandeh, Hassan. Analysis and interpretation of data: Erbulut, Zafarparandeh, Hassan. Critically revising the article: Erbulut, Zafarparandeh. Reviewed submitted version of manuscript: Erbulut, Lazoglu. Study supervision: Erbulut, Ozer.

\section{Correspondence}

Deniz U. Erbulut, Koc University, Departments of Mechanical Engineering and Neurosurgery, Rumelifeneri Yolu, 34450, Istanbul, Turkey. email: erbulutdeniz@gmail.com. 